\title{
The Representation of Contour Tones in Cantonese
}

\author{
JACKSON L. LEE \\ University of Chicago
}

\section{Introduction}

A central question in tonal phonology is the representation of tone. One of the focal points is the representation of contour tones, especially since Goldsmith (1976) and subsequent works have analyzed contour tones in Bantu languages as sequences of level tones. Cross-linguistically, it is generally well-recognized, following Yip's (1989) terminology, that contour tones in African languages are typically clusters, which are sequences of level tones and consist of multiple tonal root nodes, and that contour tones in Asian languages are typically tone units, which have only one tonal root node. This paper points out an important exception-Cantoneseparticularly in light of Yip (2001) and Barrie (2007) on Chinese contour tones. The correct view is at least implied in earlier analyses: Cantonese tones, contour and level alike, should be represented as sequences of level tones but not unitary tone units.

Recently, for Chinese languages including Cantonese, Yip (2001) and Barrie (2007) propose that the contour tone be represented as a unitary entity (i.e., a tone unit, with one tonal root node) with only the tonal onset specified. This paper argues, however, that this is incorrect for Cantonese. Specifically, the correct representation of contour tones in Cantonese is the one akin to the Africanist tradition where the tonal onset and offset are independently specified as two separate tonal root nodes. Three arguments converge to this conclusion: (i) the phonetic realization of the high-rising tone, (ii) tonal morphophonology, and (iii) the mapping between tone and musical melody in pop music.

The structure of this paper is as follows. First, section 1 introduces the proposal of Chinese contour tone representation by Yip (2001) and Barrie (2007) and its predictions. In section 3, these predictions are shown to be false when faced with Cantonese, after my proposal of tonal representation for Cantonese is presented in section 2. Finally, section 4 concludes the paper with analytical, typological, and methodological remarks. 
Jackson L. Lee

\section{The one-target contour tone representation by Yip and Barrie}

To set the stage for my reanalysis of Cantonese tonal representation, this section presents the contour tone representation for Chinese languages by Yip (2001) and Barrie (2007). The predictions of Yip and Barrie's proposal are highlighted.

\subsection{Tonal space}

The discussion of contour tone representation begins with how pitch levels are featurally represented. Following Yip (1980) and Pulleyblank (1986), the tonal space is divided into four levels with the two binary features [ \pm upper] and $[ \pm$ raised]:

(1) The four-level tonal space

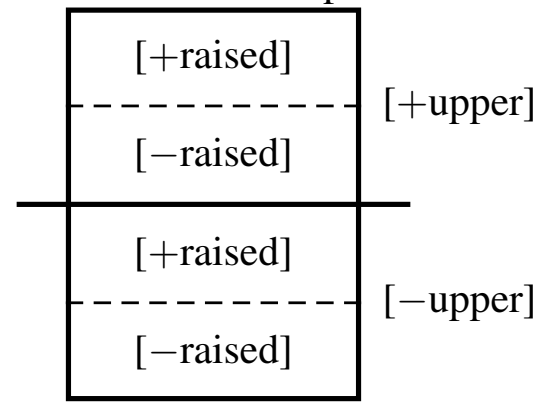

\subsection{Yip and Barrie's one-target contour tone representation}

With this categorically delineated tonal space, the next step towards a tonal representation is to ask how exactly these features are structured to represent different types of tones. To a large extent, it is this question which is at the center of this paper. For Chinese languages, Yip (2001) has recently provided an answer for contour tones; a main reason supporting her idea is the phonetics of contour tones (see section 3.1). Barrie (2007) adopts and further develops Yip's (2001) proposal. In this present paper, we focus on contour tones, though level tones will also be discussed, particularly in the analysis of Cantonese tones. The terms 'tonal onset' and 'tonal offset' are used as descriptive labels to refer to the starting and ending points of a contour tone, respectively. ${ }^{1}$

The major characteristics of Yip and Barrie's proposal for contour tones in Chinese languages are as follows. First, as is generally assumed for Chinese, contour tones are unitary entities, with only one tonal root node. Second, only one register

${ }^{1}$ Two remarks are in order. First, Yip (2001) and Barrie (2007) differ in terms of level tones; see section 2. Second, throughout this paper, the binary features [ \pm upper] and [ \pm raised], as named, are used. In fact, Barrie (2007) uses unary features [upper] and [lower] in lieu of the binary [ \pm upper], whereas Yip (2001) uses [ \pm high] instead of $[ \pm$ raised]. These terminological differences have no effect on our discussion. 
feature [ \pm upper] is specified for the whole contour tone. Third, only the tonal onset, but not tonal offset, is specified for the pitch feature [ \pm raised]. That is, this is a one-target proposal, with only the tonal onset explicitly and fully specified (cf. the two-target unitary-entity proposal in Yip (1989)). Fourth, a [contour] feature (Barrie) or an unspecified "rebound" (Yip) signals a contour tone. All these properties are illustrated by the following examples, based on Barrie's system:

(2) The one-target tone unit representation

a. High-rising tone
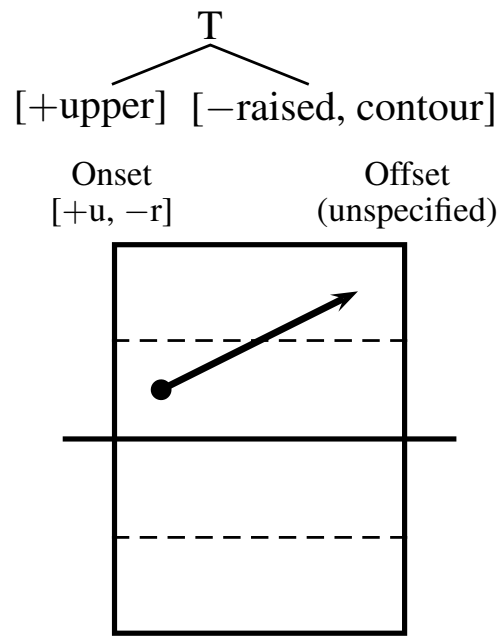

b. Mid-level tone
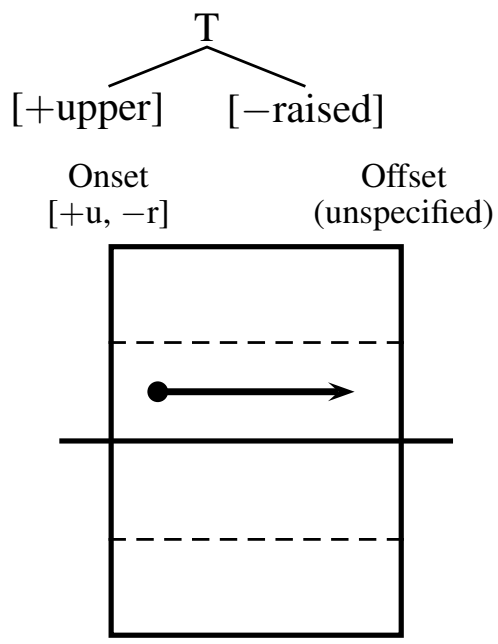

In (2) above, the two exemplified tones are a high-rising tone and a mid-level tone. In terms of the one-target tonal representations, these two tones share a great deal in common. They are both tone units with only one tonal root node denoted by ' $\mathrm{T}$ ' in (2). Their tonal onsets are the same: the register feature is [+upper] and the pitch feature is [-raised]. The only difference between the two tones is that the pitch feature of the high-rising tone (2a) has the additional specification of [contour], which requires the tonal trajectory to deviate from the tonal onset. In the case of the high-rising tone here, the tone can only go upward but not downward, because going downward would cross the boundary between [+upper] and [-upper]. This is disallowed, because the entire tone is specified to be [+upper]. Without [contour], as in (2b) for the mid-level tone, the tone simply stays level.

The two tones in (2) are also depicted using the tonal-space diagram from (1). The solid black dots $\bullet$ denote fully specified tonal targets; only the tonal onsets are specified. The arrows show the direction of tonal trajectory. The tonal offsets are featurally unspecified, but are implied by where the tonal trajectory ends.

\subsection{Predictions of Yip and Barrie's proposal}

Two important predictions stem from Yip and Barrie's proposal with respect to Chinese contour tones: 
(3) Predictions of Yip (2001) and Barrie (2007) on Chinese contour tones

a. A contour tone cannot cross the boundary between [+upper] and [-upper].

b. The tonal offset of a contour tone cannot be specifically referred to by the phonology.

Both predictions have been alluded to in the discussion of the two tones in (2) above. The first prediction, in (3a), results from the property of Yip and Barrie's proposal that only one register feature [ \pm upper] is specified for the whole contour tone. The second prediction, in (3b), is due to the fact that the tonal offset of a contour tone is not fully specified in Yip and Barrie's proposal: $[ \pm$ raised $]$ is unspecified for the tonal offset.

Both Yip (2001) and Barrie (2007) analyze the contour tones of a particular Chinese language, Cantonese, in terms of their proposal of tonal representation. In fact, however, Cantonese is a counterexample among the Chinese languages they discuss. Both predictions in (3) are false with respect to Cantonese. In the following, the analysis of Cantonese tones in Yip and Barrie's terms is contrasted with my reanalysis in section 2 . To justify the proposed reanalysis, the arguments that the predictions in (3) are falsified for Cantonese are presented in section 3.

\section{Cantonese tones: The reanalysis}

This section presents the analysis of Cantonese tones in terms of Yip (2001) and Barrie's (2007) proposal and contrasts it with my proposed reanalysis, with crucial differences in relation to the predictions in (3) above.

\subsection{Cantonese tones as one-target tone units}

Yip (2001) and Barrie's (2007) analysis of Cantonese contour tones follows their proposal of the one-target contour tone representation. Note that Yip (2001) and Barrie (2007) share a similar analysis for contour tones but differ in terms of level tones; for Yip, both tonal onset and offset of level tones are featurally specified for [ \pm upper $]$ and $[ \pm$ raised].

In (4) below, the analysis of Cantonese tones is illustrated based on Barrie's discussion, particularly the featural specifications from Barrie (2007:351). The sixway lexical tonal contrast in Cantonese is illustrated with the segmental material [si]. Following the convention in the literature on Chinese linguistics, tones are transcribed using the Chao tone numbers, where ' 5 ' denotes the highest tone and ' 1 ' the lowest. The featural specifications for [ \pm upper, \pm raised] are based on Barrie's. For clarity, I also present Barrie's analysis visually in a tonal-space diagram on the right hand side of (4). In Barrie's representations, there are two contour tones which bear [contour] featurally and therefore have a non-horizontal tonal trajectory. The 


\section{The Representation of Contour Tones in Cantonese}

features $[ \pm$ upper $]$ and $[ \pm$ raised $]$ specify only the tonal onsets.

(4) Cantonese tones as one-target tone units, based on Barrie (2007)
a. Contour tones
'history' si35
[+upper, -raised, contour]
Onset Offset
'market' si23 [-upper, -raised, contour]
b. Level tones

$\begin{array}{lll}\text { 'poem' } & \text { si55 } & \text { [ }+ \text { upper, }+ \text { raised] } \\ \text { 'to try' } & \text { si33 } & \text { [ }+ \text { upper, }- \text { raised] } \\ \text { 'affair' } & \text { si22 } & \text { [- } \text { - upper, +raised] } \\ \text { 'time' } & \text { si21 } & \text { [- } \text { - upper, - raised] }\end{array}$

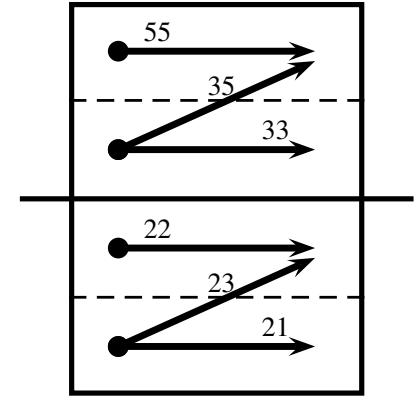

The point of presenting Barrie's analysis of Cantonese tones is to show how a particular tone system can be represented in terms of the one-target tonal representations. The way how particular tones in Cantonese are represented is not critical here. For instance, the tone 21 is sometimes transcribed as 11 due to variation in production between a low-falling tone and an extra-low level tone; see Matthews and Yip (2011:ch.1).

\subsection{The reanalysis}

In contrast to Barrie's analysis, this paper proposes that Cantonese tones, contour and level alike, be represented as sequences of level tones, each with its own tonal root node and featural specifications for [ \pm upper] and [ \pm raised]. In other words, the reanalysis is that Cantonese tones are representationally similar to those in Bantu languages. One possible way to represent a Cantonese tone geometrically is in (5) below: ${ }^{2}$

(5) Cantonese tone represented with independent tonal root nodes and features

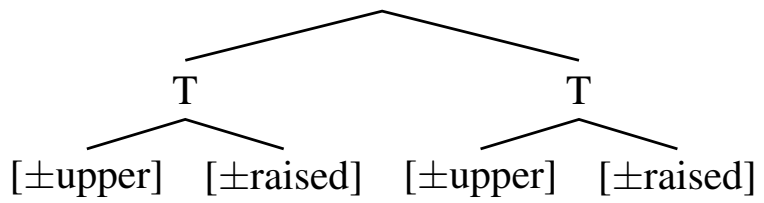

The Cantonese tone as represented in (5) above has two independent tonal root nodes, each denoted by ' $T$ '. The first ' $T$ ' on the left represents the tonal onset, and the second ' $\mathrm{T}$ ' on the right the tonal offset. Each tonal root node has its own

\footnotetext{
${ }^{2}$ Whether the register feature [ \pm upper $]$ and the pitch feature $[ \pm$ raised] are in a sisterhood relationship or some sort of dominance relationship is immaterial to the points of interest in this paper.
} 
[ \pm upper $]$ and $[ \pm$ raised $]$. Both tonal onset and offset are fully specified in featural terms.

Under this reanalysis, the six Cantonese tones are represented as follows:

(6) The six Cantonese tones as sequences of level tones

a. Featural representations:

\begin{tabular}{lccccc}
\hline \multicolumn{1}{c}{ Tone } & & \multicolumn{2}{c}{ Onset } & \multicolumn{2}{c}{ Offset } \\
& & {$[ \pm$ upper $]$} & {$[ \pm$ raised $]$} & {$[ \pm$ upper $]$} & {$[ \pm$ raised $]$} \\
\hline High-level & 55 & + & + & + & + \\
Mid-level & 33 & + & - & + & - \\
Low-level & 22 & - & + & - & + \\
\hdashline High-rising & 25 & $-*$ & + & $+*$ & + \\
Low-rising & 23 & $-*$ & + & $+*$ & - \\
Low-falling & 21 & - & + & - & - \\
\hline
\end{tabular}

b. In the tonal-space diagram:

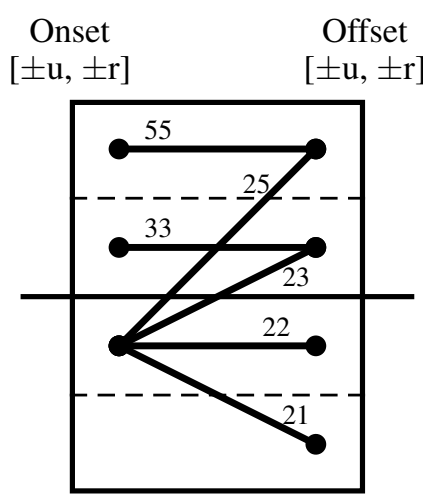

The two most important differences between my analysis of Cantonese tones and Barrie's lie in the representation of contour tones and tonal offsets. First, in my analysis, the two rising tones-high-rising 25 and low-rising 23-switch the feature value for [ \pm upper] from ' - ' at the tonal onset to ' + ' at the tonal offset, marked by ' $*$ ' in (6a). In terms of the diagram in (6b), these two tones cross the boundary separating the [+upper] and [-upper] regions, contra the prediction of Yip and Barrie that a tone can only be within either [+upper] or [-upper] (3a). Second, the tonal offsets of all tones, contour and level alike, are fully specified for both [ \pm upper] and [ \pm raised]. This is indicated by the solid black dots for tonal offsets in (6b); there are no arrows in this diagram, cf. (4) above. The theoretical implication is that, contrary to the prediction in $(3 \mathrm{~b})$, phonology can potentially refer to tonal offsets.

Although this reanalysis with sequences of level tones is arguably less parsimonious than Barrie's analysis using one-target tone units, the next section justifies my analytical choice with empirical evidence. 


\section{The Representation of Contour Tones in Cantonese}

\section{The arguments}

This section presents the arguments that Cantonese tones should be represented as sequences of level tones rather than tone units. The arguments are from the perspectives of (i) the phonetic realization of the high-rising tone, (ii) tonal morphophonology, and (iii) the mapping between tone and musical melody in pop music.

\subsection{Argument 1: Phonetic realization of the high-rising tone}

The first argument aims at a better phonetics-phonology mapping in general, a goal which is also endorsed by Yip (2001) herself. Specifically, the argument is about the phonetics of the Cantonese high-rising tone. In Cantonese, there are two sources of the high-rising tone: it can either be a lexical high-rising tone or a derived highrising tone from another tone. The complete argument here has to do with both of these two types of the high-rising tone.

\subsubsection{The lexical high-rising tone}

Bauer and Benedict (1997) report that the lexical high-rising tone, commonly transcribed as 35 in and before the 1990s (see, e.g., Yue-Hashimoto (1972); Matthews and Yip (1994)), begins acoustically at a similar pitch level as the low-rising tone 23. The high-rising tone has a lower acoustic tonal onset than previously thought. Moreover, the mid-level tone 33 has an acoustic tonal onset higher than that of the high-rising tone and the low-rising tone 23. All these instrumental observations led Bauer and Benedict (1997) to suggest a revision of the transcription convention: the (lexical) high-rising tone is more suitably transcribed as 25 . The following pitch tracks from Mok and Wong (2010) show that the high-rising tone 25 shares a similar tonal onset with the low-rising tone 23 but not with the mid-level tone 33 .

(7) Pitch tracks of Cantonese tones from Mok and Wong (2010), with tone numbers added

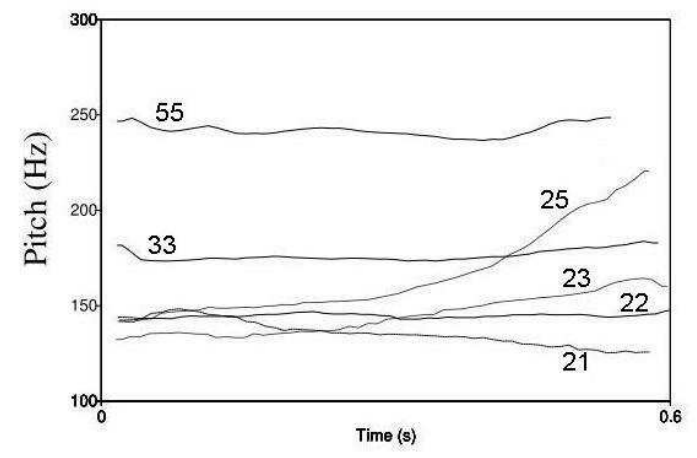


The phonetics of the high-rising tone has important implications for its phonological representation. If the mid-level 33 is in the [+upper] region, and if the lowrising tone 23 has its tonal onset as [-upper], then the high-rising tone 25 should begin as [-upper] as the 23 tone does. However, the 25 tone clearly must end with [+upper] for its tonal offset. In other words, the high-rising tone 25 crosses the boundary between [-upper] and [+upper]. This is a paradox for Yip and barrie's one-target contour tone unit proposal, because it allows a tone to be specified for only one, but not two, [ \pm upper] feature. The first prediction of their proposal, from (3), that a contour tone cannot straddle the [ \pm upper] boundary, is falsified.

\subsubsection{The derived high-rising tone}

The Cantonese high-rising tone can be derived by a (possibly optional) tonealternating process for a variety of semantic and morphological reasons. This is illustrated in (8) below, where the tones are transcribed in the way this paper ultimately intends; ' $\underline{\mathbf{5}}$ ' denotes a derived high tone ' 5 '. Such tonal alternation applies to all tones as input, except for the high-level 55 and the high-rising 25 itself.

(8) Cantonese tonal alternation

(cf. Bauer and Benedict 1997; Matthews and Yip 2011; Yu 2007a,b; YueHashimoto 1972)
a. Nominalization

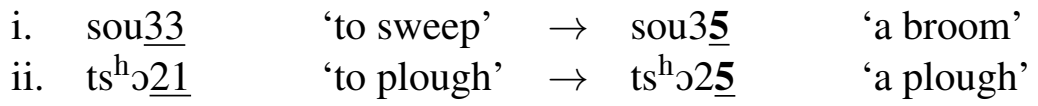
b. Aspects (e.g., perfective)
i. $\operatorname{sik} \underline{22}$ tso25 'eat-PERF' $\sim \operatorname{sik} 2 \underline{5}$ 'eat.PERF'
ii. $\operatorname{kin} 3 \underline{3}$ tso25 'see-PERF' $\sim \operatorname{kin} 3 \underline{5} \sim$ 'see.PERF'
c. Unpredictable/no apparent meaning
i. wu21 tip22 'butterfly' $\sim$ wu21 tip2 $\underline{5}$ 'butterfly'

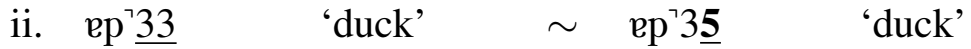

$\mathrm{Yu}$ (2007a) discovers that, if the non-derived tone is the mid-level 33 (but not 23,22 , or 21 with an acoustically lower tonal onset), the derived high-rising tone actually has a correspondingly higher acoustic tonal onset than the lexical high-rising tone. Also, if the non-derived tone is one of 23, 22, and 21 (but not 33), the derived high-rising tone is acoustically indistinguishable from the lexical high-rising 25 . This means that there are two distinct high-rising tones. We shall transcribe the one derived from 33 as 35, and the one derived from 23, 22, and 21 as 25.

In terms of their phonological representations, the two high-rising tones 25 and 35 pose a problem to Yip and Barrie's one-target contour tone representaion. Both 25 and 35 must end in [+upper] for their tonal offset. If 35 is entirely within the [+upper] tonal space, then the only way to represent another rising tone ending in [+upper] is to say that the tonal onset of 25 is [-upper]. This is disallowed in 


\section{The Representation of Contour Tones in Cantonese}

Yip and Barrie's system, however. If the two high-rising tones 25 and 35 are to be featurally represented by $[ \pm$ upper] and $[ \pm$ raised], then the prediction that a contour tone can only be either [+upper] or [-upper], from (3), does not hold.

For Yip (2001), one important motivation for the one-target tone unit proposal is the phonetics of contour tones. She points out that, phonetically, the contour tones in Chinese languages mostly begin as a level plateau as the tonal onset and simply shoot away from the tonal onset without an ending level plateau. The tonal offset, then, is wherever the tonal trajectory ends. This is why Yip argues that only the tonal onset of a contour tone is featurally fully specified and that some other feature signals the drift of the pitch away from the tonal onset. To the extent that Yip's idea works for numerous cases of Chinese contour tones, it does not properly capture the rather complex situation of Cantonese contour tones, as discussed in this section.

\subsection{Argument 2: Tonal morphophonology}

Barrie (2007) extends Yip's (2001) proposal of one-target contour tone representation to include level tones as well. That is, for Barrie (2007), generally all Chinese tones, contour and level alike, are represented as one-target tone units. This presents a problem to the tonal morphophonological analysis of Cantonese. Having the tonal onset and offset independently and fully specified for [ \pm upper] and [ \pm raised] eliminates the problem.

We have actually examined some of the important data. In Cantonese, the only synchronically productive (morpho)phonological process is the kind of tonal alternation illustrated in (8) above, where a high-rising tone is derived from another tone. The new data presented below is the attenuatives (meaning 'a little X') in Cantonese which demonstrate the points to be made in this section most succinctly.

(9) Cantonese attenuatives (Lee 2012)
a. syn55 'sour' $\rightarrow$ syn55 syn5 $\underline{5}$ ter25
b. jiu25 'girly' $\rightarrow$ jiu25 jiu2 $\underline{\mathbf{5}}$ ter 25
c. ts ${ }^{\mathrm{h}} \mathrm{i} 33$ 'similar' $\rightarrow$ ts $^{\mathrm{h}} \mathrm{i} 33$ ts ${ }^{\mathrm{h}} \mathrm{i} 3 \underline{\mathbf{5}}$ ter 25
d. kuy22 'tired' $\rightarrow$ kuy22 kuy $2 \underline{5}$ ter25
e. $\mathrm{k}^{\mathrm{h}}$ en23 'near' $\rightarrow \mathrm{k}^{\mathrm{h}} \mathrm{en} 23 \mathrm{k}^{\mathrm{h}} \mathrm{en} 2 \underline{5}$ ter 25
f. hon 21 'red' $\rightarrow$ hon 21 hon $2 \underline{5}$ ter 25

In (9), each of the six tones in Cantonese is illustrated with a word and its attenuative. The attenuative constructions involve reduplication and the concomitant affix ter25. Of particular interest is the tone of the second reduplicant. Ostensibly, only the non-derived tones $33,22,23$, and 21 undergo tonal alternation to become a high-rising tone ( $9 c-f)$, but the tones 55 and 25 remain unaltered (9a-b). A more insightful way to describe this situation is to say that the tonal offset of a non-derived tone (any of the six lexical tones) has to be the high tone ' 5 ' at the second reduplicant. Under this view, there is tonal alternation at the analytical level for the 
non-derived high-level 55 and high-rising 25, although on the surface it appears that nothing happens. Tonal alternation applies vacuously for 55 and 25 .

A straightforward analysis to capture such tonal behavior for Cantonese has been around for a long while, at least since Yip (1980). The formal analysis hinges on a floating high tone which is diachronically motivated; see Yu (2007b) for the historical details. In brief, it consists of the docking of the floating tone and the delinking of some other tonal specification, as illustrated with the non-derived lowfalling tone 21 in (10) here:

(10) The floating-tone analysis of Cantonese tonal alternation (Yip 1980; Cheung 1986; Bao 1999; Chen 2000)

a.

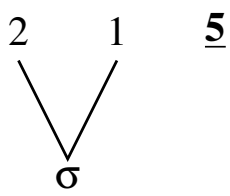

b.

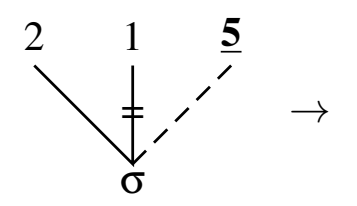

c.

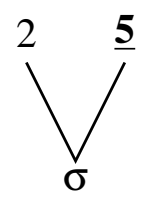

This floating-tone analysis entails that only tonal offsets are altered in tonal alternation, and that the tonal onsets are unaffected. This is empirically supported by the phonetics of the derived high-rising tone as discussed in section 3.1 above.

The phonological representations of Cantonese tones should readily accommodate such an analysis of tonal alternation. If the tonal offsets are fully specified for [ \pm upper] and [ \pm raised], as is argued in this paper, then the floating-tone analysis amounts to delinking the tonal root node of the tonal offset, with replacement by a high-tone offset ' 5 ' specified as [+upper] and [+raised]. In contrast, Yip and Barrie's representations of Cantonese contour tones would not allow this, because the tonal offsets of contour tones are not fully specified and consequently there is no tonal offset to delink. Yip and Barrie's analysis of Cantonese contour tones is incompatible with the floating-tone analysis of Cantonese tonal alternation in (10).

For complete argumentation, the following question is in order: how would tonal alternation in (9) be accounted for if Cantonese tones were represented as one-target tone units? We use Barrie's analysis of Cantonese tones for discussion (Yip does not analyze level tones as one-target tone units):

(11) Cantonese tonal alternation in terms of Barrie's tonal representations

a. One-target input tones

\begin{tabular}{llll}
\hline & {$[ \pm \mathrm{u}]$} & {$[ \pm \mathrm{r}]$} & [contour] \\
\hline 55 & + & + & $\varnothing$ \\
33 & + & - & $\varnothing$ \\
22 & - & + & $\varnothing$ \\
35 & + & - & $\checkmark$ \\
23 & - & - & $\checkmark$ \\
21 & - & - & $\varnothing$ \\
\hline
\end{tabular}

b. What the outputs would be

\begin{tabular}{llll}
\hline & {$[ \pm \mathrm{u}]$} & {$[ \pm \mathrm{r}]$} & {$[$ contour] } \\
\hline 55 & + & + & $\emptyset$ \\
35 & + & - & $\checkmark *$ \\
35 & $+*$ & $-*$ & $\checkmark *$ \\
35 & + & - & $\checkmark$ \\
35 & $+*$ & - & $\checkmark$ \\
35 & $+*$ & - & $\checkmark *$ \\
\hline
\end{tabular}




\section{The Representation of Contour Tones in Cantonese}

(11a) is Barrie's analysis of Cantonese tones, identical to (4). For [contour], since it is a unary feature, ' $\checkmark$ ' denotes its presence, and ' $\varnothing$ ' its absence. (11b) is what Barrie's analysis would have to say as to what the six tones become for tonal alternation in Cantonese attenuatives in (9). The high-level 55 and high-rising 35 remain changed, whereas all other tones become the high-rising 35 . Interestingly, Barrie is perfectly aware of the Cantonese attenuative data in (9), as is clear from his footnote 20 on page 350 , but no analysis is provided.

In terms of Barrie's representations, a comparison between (11a) and (11b) reveals what features would have to be altered. In (11b), the features marked by '*' are different from their counterparts in (11a), which do not appear to pattern together in a consistent pattern. If the goal of a phonological analysis is to come up with a unified analysis for the tonal (non-)alternation involved, then it is unclear how the features which would be altered can be described by natural classes. For this reason, representing Cantonese tones as sequences of level tones is a preferred option, so that the floating-tone analysis of tonal alternation can be expressed directly to account for the attenuative reduplication data.

The upshot of this argument is that Cantonese contour and level tones bear fully specified tonal offsets because they are sequences of level tones rather than onetarget tone units. The floating-tone analysis of tonal alternation crucially makes reference to tonal offsets. The second prediction from Yip (2001) and Barrie's (2007) proposal that the tonal offset of a contour tone cannot be referred to by phonology, stated in (3b), is falsified.

\subsection{Argument 3: Mapping between tone and musical melody in pop music}

The third argument is about the mapping between tone and musical melody in Cantonese pop music, given that songs shed light on phonological theory; see, for instance, Dell (2011) on Berber syllables. Phonologists study songs sung in tone languages because of the intriguing questions of how the tones of the lyrics map onto the musical melody and what the mapping patterns or restrictions are, if any. For Cantonese songs, it is the tonal offsets which play an important role, which suggests that they are phonologically specified.

Researchers have observed that, in Cantonese lyrics writing for pop music, the low-rising 23 and mid-level 33 are interchangeable, whereas the high-rising 25 and the high-level 55 are interchangeable (Chan 1987; Ho 2006). The tones are interchangeable in the sense that, for example, if the tone 23 is compatible with a particular musical note, then 33 is also compatible; see the references cited for more musical details. The consensus is that the tone pairs 55/25 and 33/23 pattern in the way they do by sharing the same tonal offset. If this is the case, then the phonological representations of the tonal offsets should reflect that they are explicitly referred to. The implication is that Cantonese tones are sequences of level tones, so that the tonal offsets are fully specified and can be phonologically referred to. 
In the following, we compare how the tone pairs 55/25 and 33/23 are represented by phonological features in two systems, the one-target tone unit system by Barrie (2007) and my proposal with sequences of level tones.

(12) Comparing the featural specifications of the tone pairs 55/25 and 33/23 a. Cantonese tones à la Barrie (2007)

b. The reanalysis in this paper

\begin{tabular}{c:c:c:c} 
& {$[ \pm \mathrm{u}]$} & {$[ \pm \mathrm{r}]$} & [contour] \\
\hline 55 & $+{ }^{*}$ & + & $\varnothing$ \\
35 & $+*$ & - & $\checkmark$ \\
33 & + & $-*$ & $\varnothing$ \\
23 & - & $-*$ & $\checkmark$ \\
21 & - & - & $\varnothing$ \\
22 & - & + & $\varnothing$ \\
\hline
\end{tabular}

\begin{tabular}{ccc:cc}
\hline & \multicolumn{2}{c}{ Onset } & \multicolumn{2}{c}{ Offset } \\
& {$[ \pm \mathrm{u}]$} & {$[ \pm \mathrm{r}]$} & {$[ \pm \mathrm{u}]$} & {$[ \pm \mathrm{r}]$} \\
\hline 55 & + & + & + & + \\
25 & - & + & + & + \\
33 & + & - & + & - \\
23 & - & + & + & - \\
21 & - & + & - & - \\
22 & - & + & - & + \\
\hline
\end{tabular}

The point is that each of the tone pairs 55/25 and 33/23 should be uniquely identified in featural terms. Barrie's (2007) representational system in (12a) is unable to do so for neither of the tone pairs. Although 55 and 35 pattern together by [+upper] only (marked by '*' in (12a)), 33 is also [+upper]; the relevant feature values are enclosed in a dotted box in (12a). A similar issue arises for the tone pair 33/23: both 33 and 23 share [-raised] only (marked by ' ${ }^{*}$ '), but 35 and 11 are also [-raised]. In contrast, the reanalysis of Cantonese tones in this paper squarely captures the two tone pairs straightforwardly, as shown by the dotted boxes in (12b), because the tonal offsets are fully specified. It is noteworthy that Yip (2001) also attempts to explain the Cantonese tone-music mapping patterns discussed in this section, albeit with ad hoc formalism.

As in the second argument on tonal morphophonology in section 3.2 above, this argument on tone-music mapping shows that the tonal offsets in Cantonese have to be referred to by phonology and specified separately from tonal onsets, thus falsifying the prediction in (3b) that phonology cannot refer to tonal offsets.

\section{Conclusions}

This paper has argued that, with a focus on contour tones, Cantonese tones are represented as sequences of level tones, rather than unitary entities. If phonology is an abstract representational system, then this paper has pushed for a closer mapping between phonology and other domains: (i) phonetics, for the tonal trajectory of the high-rising tone; (ii) morphology, for the attenuative and other derived constructions; and (iii) native-speaker intuition, for how tones are categorized as evidenced by songs. It is reasonable to ask if an analysis with sequences of level tones rather than one-target tone units is less parsimonious. Indeed, the answer is positive: if both tonal onsets and offsets have their own independent [ \pm upper] and 


\section{The Representation of Contour Tones in Cantonese}

[ \pm raised], then a tonal inventory of 16 tones is predicted, but Cantonese has only six tones. Notwithstanding this mathematical issue, there are good reasons to opt for sequences of level tones for Cantonese. First, if we stick to binary features only, there are no feature systems that could single out six distinct objects; two features would differentiate four objects, and three features eight. Second, phonological features are meant to be interpretable. If an $n$-ary or hybrid feature system is able to represent exactly six entities, it is unclear if the features involved in such a system are sufficiently meaningful; for instance, one single senary feature with six possible values might well capture the six Cantonese tones, but such a feature is inevitably meaningless.

Typologically, Cantonese joins other Chinese varieties whose contour tones have been argued to not be represented as contour tone units; see Duanmu (1994) and Chen (2010). Beyond the Sinitic family but still within Asia, tone languages without unitary contour tone units are not unheard of, an example being KukiThaadow (Hyman 2007). This present paper further undermines the general view that contour tones across African and Asian languages are fundamentally different in terms of representation.

From the methodological perspective, this paper has demonstrated that it is important to analyze individual languages solo without the assumption that genetically related languages (e.g., Chinese languages) somehow share the same grammar. In Chinese linguistics, there is sometimes a controversial assumption of a universal Chinese grammar, at least implicitly (Yue-Hashimoto 1993; Matthews 1999, To appear). A typical scenario has a few Chinese varieties examined for a given research question, and then the conclusions are generalized to the entire Chinese group. This paper argues for a more cautious approach. Analytically, this paper is concerned solely with Cantonese. As such, all conclusions drawn only apply to Cantonese and not necessarily, and certainly not immediately, to any other Chinese languages, or other languages in general. It is after individual linguistic varieties are analyzed that we make concrete typological statements for the varieties studied.

\section{Acknowledgments}

I am grateful for the comments from and discussion with Yuni Kim, Stephen Matthews, Jason Riggle, and Alan Yu. Thanks are also due to the organizers and au-

diences at the $38^{\text {th }}$ Annual Meeting of the Berkeley Linguistics Society in February 2012, the Workshop on Innovations in Cantonese Linguistics at the Ohio State University in March 2012, and the $36^{\text {th }}$ Penn Linguistics Colloquium in March 2012. I also thank François Dell, a conversation with whom has ultimately prompted me to put together this paper. 
Jackson L. Lee

\section{References}

Bao, Zhiming. 1999. The Structure of Tone. Oxford: Oxford University Press.

Barrie, Michael. 2007. Contour tones and contrast in Chinese languages. Journal of East Asian Linguistics 16:337-362.

Bauer, Robert S. and Paul K. Benedict. 1997. Modern Cantonese Phonology. Berlin: Mouton de Gruyter.

Chan, Marjorie. 1987. Tone and melody in Cantonese. In Proceedings of the Thirteenth Annual Meeting of the Berkeley Linguistics Society, 26-37.

Chen, Matthew. 2000. Tone Sandhi: Patterns Across Chinese Dialects. Cambridge, UK: Cambridge University Press.

Chen, Tsung-Ying. 2010. Some remarks on Contour Tone Units. Journal of East Asian Linguistics 19(2):103-135.

Cheung, Kwan-Hin. 1986. The Phonology of Present-day Cantonese. Doctoral dissertation, University College, London.

Dell, François. 2011. Singing in Tashlhiyt Berber, a language that allows vowel-less syllables. In Charles E. Cairns and Eric Raimy, eds., Handbook of the Syllable, Leiden: Brill.

Duanmu, San. 1994. Against contour tone units. Linguistic Inquiry 25(4):555-608.

Goldsmith, John A. 1976. Autosegmental phonology. Doctoral dissertation, MIT.

Ho, Wing See Vincie. 2006. The tone-melody interface of popular songs written in tone languages. In M. Baroni, A. R. Addessi, R. Caterina, and M. Costa, eds., Proceedings of the 9th International Conference on Music Perception and Cognition (ICMPC9), 1414-1422, The Society for Music Perception and Cognition and European Society for the Cognitive Sciences of Music.

Hyman, Larry M. 2007. Kuki-Thaadow: An African tone system in Southeast Asia. In UC Berkeley Phonology Lab Annual Report (2007).

Lee, Jackson L. 2012. Fixed-tone reduplication in Cantonese. In McGill Working Papers in Linguistics 22(1). Proceedings from the Montreal-Ottawa-Toronto (MOT) Phonology Workshop 2011: Phonology in the 21st Century: In Honour of Glyne Piggott.

Matthews, Stephen. 1999. Y. R. Chao and Universal Chinese Grammar. In David Cram, Andrew Robert Linn, and Elke Nowak, eds., History of Linguistics 1996, 217-224, Amsterdam: John Benjamins. 
Matthews, Stephen. To appear. Is there a universal Chinese grammar? In Proceedings of the 24th North American Conference on Chinese Linguistics.

Matthews, Stephen and Virginia Yip. 1994. Cantonese: A Comprehensive Grammar. London: Routledge, 1st edition.

Matthews, Stephen and Virginia Yip. 2011. Cantonese: A Comprehensive Grammar. London: Routledge, 2nd edition.

Mok, Peggy and Peggy Wong. 2010. Production of the merging tones in Hong Kong Cantonese: preliminary data on monosyllables. In Proceedings of Speech Prosody 2010, Chicago, IL.

Pulleyblank, Douglas. 1986. Tone in Lexical Phonology. Dordrecht: D. Reidel.

Yip, Moira. 1980. The tonal phonology of Chinese. Doctoral dissertation, MIT.

Yip, Moira. 1989. Contour tones. Phonology 6(1):149-174.

Yip, Moira. 2001. Tonal features, tonal inventories and phonetic targets. UCL Working Papers in Linguistics 161-188.

Yu, Alan C. L. 2007a. Tonal phonetic analogy. Proceedings of the International Congress of Phonetic Sciences XVI 1749-1752.

Yu, Alan C. L. 2007b. Understanding near mergers: the case of morphological tone in Cantonese. Phonology 24:187-214.

Yue-Hashimoto, Anne Oi-Kan. 1972. Studies in Yue Dialects (vol. 1): The Phonology of Cantonese. Cambridge: Cambridge University Press.

Yue-Hashimoto, Anne Oi-Kan. 1993. Comparative Chinese Dialectal Grammar: Handbook for Investigators. Paris: Centre de Recherches Linguistiques sur l'Asie Orientale.

Jackson L. Lee

Department of Linguistics

University of Chicago

1010 East $59^{\text {th }}$ Street

Chicago, IL 60637

jsllee@uchicago.edu 